\title{
Inhibition of APN/CDI 3 leads to suppressed progressive potential in ovarian carcinoma cells
}

\author{
Mikio Terauchi, Hiroaki Kajiyama*, Kiyosumi Shibata, Kazuhiko Ino, \\ Akihiro Nawa, Shigehiko Mizutani and Fumitaka Kikkawa
}

\begin{abstract}
Address: Department of Obstetrics and Gynecology, Nagoya University, Graduate School of Medicine, Tsuruma-cho 65, Showa-ku, Nagoya 4668550, Japan

Email: Mikio Terauchi - mikio727@med.nagoya-u.ac.jp; Hiroaki Kajiyama* - kajiyama@med.nagoya-u.ac.jp;

Kiyosumi Shibata - shiba@med.nagoya-u.ac.jp; Kazuhiko Ino - kazuino@med.nagoya-u.ac.jp; Akihiro Nawa - nawa2005@med.nagoya-u.ac.jp; Shigehiko Mizutani - shigehiko-m@proof.ocn.ne.jp; Fumitaka Kikkawa - kikkawaf@med.nagoya-u.ac.jp

* Corresponding author
\end{abstract}

Published: 27 July 2007

BMC Cancer 2007, 7:140 doi:10.1186/147/-2407-7-140
Received: 17 August 2006

Accepted: 27 July 2007

This article is available from: http://www.biomedcentral.com/I47/-2407/7//40

(C) 2007 Terauchi et al; licensee BioMed Central Ltd.

This is an Open Access article distributed under the terms of the Creative Commons Attribution License (http://creativecommons.org/licenses/by/2.0), which permits unrestricted use, distribution, and reproduction in any medium, provided the original work is properly cited.

\begin{abstract}
Background: Aminopeptidase N (APN/CDI3), a 150-kDa metalloprotease, is a multifunctional cell surface aminopeptidase with ubiquitous expression. Recent studies have suggested that APN/ CDI3 plays an important role in tumor progression of several human malignancies. In the current study, we investigated the role of APN/CDI 3 in ovarian carcinoma (OVCA) progression.
\end{abstract}

Methods: We first examined the expression of APN/CDI3 at the protein level in a variety of OVCA cell lines and tissues. We subsequently investigated whether there was a correlation between APN/CDI 3 expression and invasive potential of various OVCA cell lines. Moreover, we investigated the function of APN/CDI3 in OVCA cells using bestatin, an APN/CDI3 inhibitor, or transfection of siRNA for APN/CDI3.

Results: We confirmed that APN/CDI 3 was expressed in OVCA tissues and cell lines to various extents. There was a positive correlation between APN/CDI3 expression and migratory potential in various OVCA cell lines with accordingly enhanced secretion of endogenous MMP-2. Subsequently, we found a significant decrease in the proliferative and migratory abilities of OVCA cells after the addition of bestatin or the inhibition of APN/CDI3 expression by siRNA. Furthermore, in an animal model, daily intraperitoneal administration of bestatin after inoculation of OVCA cells resulted in a decrease of peritoneal dissemination and in prolonged survival of nude mice.

Conclusion: The current data indicate the possible involvement of APN/CDI3 in the development of OVCA, and suggest that clinical use of bestatin may contribute to better prognosis for ovarian carcinoma patients.

\section{Background}

Aminopeptidase N (APN/EC 3.4.11.2) is a type II membrane-bound metalloproteinase expressed on various cell types, such as kidney, intestinal epithelium, liver, placenta, and lung cells [1-3]. APN is also a cell surface aminopeptidase that was originally characterized as a myeloid 
marker[4]. APN/CD13 activates or inactivates bioactive peptides on the cell surface by cleaving them enzymatically and regulates their availability to adjacent cells. Importantly, recent reports have indicated that APN/ CD13 has a variety of functions, including roles in inflammatory and immunological responses, signal transduction, antigen processing, neuropeptide and cytokine degradation, and extracellular matrix degradation [5-9]. In addition, a number of studies have provided evidence that APN/CD13 may play a role in tumor progression by regulating processes such as cell-cell contact, proliferation, tumor invasion, and angiogenesis [5,10-14]. Furthermore, a recent study showed that APN/CD13 was involved in the protection of leukemic cells against apoptosis[15].

Epithelial ovarian carcinoma (OVCA) is a major cause of death among gynecological malignancies [16]. Since OVCA frequently remains clinically silent, the majority of patients with this disease have advanced intraperitoneal metastatic disease at diagnosis [17]. The biological behavior of this carcinoma is associated with clinicopathological parameters, including International Federation of Gynecologists and Obstetricians (FIGO) stage, tumor grade, and histological type. Treatment for advanced OVCA is difficult because of both the inability to completely resect diffuse tumors on the peritoneal surface and the eventual resistance of the tumor cells to chemotherapy.

We have investigated the molecular mechanism of OVCA progression. Especially, our recent reports focused on the involvement of cell surface aminopeptidases such as dipeptidyl peptidase IV (DPPIV/CD26) and neutral endopeptidase 24.11 (NEP/CD10) in the peritoneal progression of this carcinoma, and demonstrated that overexpression of DPPIV or NEP in highly invasive OVCA cells significantly decreased peritoneal dissemination and increased survival time in a mouse model $[18,19]$.

In the current study, we investigated the possible role of APN/CD13 in OVCA progression. We first examined the expression level of APN/CD13 in various OVCA cell lines. Subsequently, to clarify the cellular roles of APN/CD13 in OVCA, we investigated the progression of OVCA in vitro and in vivo using bestatin, an APN/CD13 inhibitor, or siRNA specific for APN/CD13. The possible function of this enzyme as an inducer of OVCA progression is proposed.

\section{Methods \\ Cell culture}

Seven human OVCA cell lines (SKOV-3, HRA, ES-2, HEY, NOS2, NOS4, and TAOV) were cultured and maintained as described previously [19]. ES-2 and HEY cells were pur- chased from the American Type Culture Collection (ATCC) and were maintained in RPMI-1640 (Sigma) supplemented with $10 \%$ fetal calf serum (FCS) and penicillin-streptomycin. These cells were incubated at $37^{\circ} \mathrm{C}$ in a humidified atmosphere containing $5 \% \mathrm{CO}_{2}$.

\section{Enzyme activity assay}

APN/CD13 enzyme activity was measured spectrophotometrically using L-leucine-p-nitroanilide (Peptide Institute, Inc.) as an APN/CD13 substrate. Whole-cell suspensions were prepared in test tubes, and then washed with phosphate-buffered saline (PBS). Thereafter, $5 \times 10^{5}$ cells were resuspended in $200 \mu \mathrm{l}$ of PBS in each well of a 96-well microtiter plate, and the substrate was added (final $1.6 \mathrm{mM}$ ). APN/CD13 enzyme activity was estimated by measuring the absorbance at $405 \mathrm{~nm}$ using a microplate reader (Labsystems, Multiskan Bichromatic) every 15 min during incubation at $37^{\circ} \mathrm{C}$.

\section{Flow cytometric analysis}

Fluorescence-activated cell sorting (FACS) was performed to quantify the expression level of APN/CD13 on the cell surface of OVCA cells. Then, the cells were incubated with phycoerythrin-conjugated monoclonal antibody specific for APN/CD13 (BD Pharmingen, CD13mAb clone: WM15, San Diego, CA) for $30 \mathrm{~min}$ at $4^{\circ} \mathrm{C}$, and washed three times with PBS. FACS data were acquired on a FACS Calibur (Becton Dickinson, San Jose, CA), and analyzed using CELL Quest software (Becton Dickinson).

\section{Immunohistochemical staining}

Fourteen tissue samples of OVCA were obtained with informed consent from patients who were surgically treated at Nagoya University Hospital. All samples were fixed in 10 percent formalin and embedded in paraffin, and sections were cut at a thickness of $4 \mu \mathrm{m}$. For heatinduced epitope retrieval, deparaffinized sections in 0.01 $\mathrm{M}$ citrate buffer were treated three times for $5 \mathrm{~min}$ at $90^{\circ} \mathrm{C}$ at $750 \mathrm{~W}$ using a microwave oven. Immunohistochemical staining was performed using the avidin-biotin immunoperoxidase technique (Histofine SAB-PO kit, Nichirei, Tokyo, Japan). Endogenous peroxidase activity was blocked by incubation with $3 \% \mathrm{H}_{2} \mathrm{O}_{2}$, and non-specific immunoglobulin binding was blocked by incubation with $10 \%$ normal rabbit serum. As a first antibody for APN/CD13 staining, anti-APN/CD13 mAb (Novocastra, CD13mAb clone: 38C12, Newcastle, United Kingdom) was used at dilution of 1:100.

\section{Inhibition of $A P N / C D / 3$ by small interfering RNA}

We designed and purchased two different siRNA duplexes of APN/CD13, si-CD13 (sense, 5'-CACCUUGGACCAAAGUAAA-3'), and si-CD13-2 (sense, 5'GAAAUGCCACACUGGUCAA-3') from Qiagen (Tokyo, Japan). Nonspecific control siRNA duplexes (si-cont) 
(sense, 5'-CUGGAUUGUAGGAAGUACCTT-3') with the same GC content as APN/CD13 siRNA were purchased from Takara (Tokyo, Japan). The siRNA was transfected into ES-2 cells at a final concentration of $80 \mathrm{nmon} / \mathrm{L}$ using a GenePorter-2 (Genlantis, San Diego, CA) according to the manufacturer's protocol.

\section{Cell doubling time}

Cells growing in log phase were harvested and plated in a 6 -well plate at $7.5 \times 10^{4}$ cells/well in RPMI1640 +10\% FCS. The number of cells was counted at 24,48, 72, and $96 \mathrm{~h}$. The cell doubling time in log phase was determined in three separate experiments.

\section{Cytotoxicity assay: trypan blue dye-exclusion test}

The trypan blue dye-exclusion test was used to determine drug-mediated cytotoxicity as described previously[20]. The following preparations were assessed for their ability to inhibit cell growth. Briefly, target tumor cells were resuspended in medium at $25 \times 10^{4}$ cells/mL after verifying cell viability by the trypan blue dye exclusion test (Sigma Chemical Co.). First, cells were incubated for $24 \mathrm{~h}$ at $25 \times 10^{4}$ cells/well using 6 -well, flat-bottomed plates. Then, various concentrations of drug samples were added for a further $24 \mathrm{~h}$. Each plate was incubated for $72 \mathrm{~h}$ at $37^{\circ} \mathrm{C}$ in a $5 \% \mathrm{CO}_{2}$ atmosphere. Following the incubations, $100 \mu \mathrm{L}$ of the trypan blue dye was added to $100 \mu \mathrm{L}$ of cell suspension, and viable and dead cells were counted. To estimate the cytotoxicity, control cell groups without any drug treatment were compared with drugtreated cell groups, and the experiments were repeated three times.

\section{Cell proliferation assay}

The cell proliferation assay was performed as described previously [18]. Cells were seeded in triplicate in 96-well plates at a density of 2000 cells in a volume of $200 \mu \mathrm{l}$ of RPMI1640 containing 10\% FCS, and cultured for 1 to 4 days in the presence or absence of bestatin (Nihon-Kayaku, Tokyo, Japan). Cell viability was assayed by a modified tetrazolium salt 3-(4,5-dimethylthiazol-2-yl)-2,5diphenyltetrazolium bromide assay using a Cell Titer 96 Aqueous One Solution Cell Proliferation Assay kit (Promega Corp., Tokyo, Japan) according to the manufacturer's instructions. Absorbance was measured at $492 \mathrm{~nm}$ using a microplate reader (Multiskan Bichromatic; Labsystems Helsinki, Finland). Furthermore, we examined the effect of siRNA transfection on the proliferation of ES-2 cells. ES- 2 cells were seeded in 96-well plates at 1500 cells/ well in $200 \mu \mathrm{l}$ of culture medium containing 10\% FCS. After 24 hours, the medium was replaced by fresh RPMI 1640 containing 5\% FCS and transfection of the siRNA duplexes (si-cont and si-CD13) was performed in same the 96-well plate using a GenePorter-2 (Genlantis, San
Diego, CA). Cell proliferation was then evaluated using a 5-day modified MTT assay as described above.

\section{In vitro migration assay}

Cell migration was assayed in 24-well Transwell cell culture chambers (Costar). Cells were suspended in the upper chamber at a final concentration of $50 \times 10^{4} / \mathrm{ml}$ in $200 \mu \mathrm{l}$ of RPMI 1640 in the presence or absence of bestatin $(0,10,100$, or $200 \mu \mathrm{g} / \mathrm{ml})$. The lower chamber contained $700 \mu \mathrm{l}$ of RPMI 1640 supplemented with 10\% FCS. After 8 hour of incubation, the tumor cells remaining on the upper surface of the filters were removed by wiping with cotton swabs, and the migrated cells on the lower surface were stained with May-Grünwald Giemsa staining. The number of cells on the lower surface of the filters was counted under a microscope at a magnification of 200. In addition, we examined the effect of siRNA transfection on the migration of ES- 2 cells. ES- 2 cells were seeded in 10cm dishes in RPMI 1640 containing 10\% FCS. After reaching 50\% confluency, the medium was replaced by fresh RPMI 1640 containing 10\% FCS, and transfection of siRNA (si-cont or si-CD13) was performed using a GenePorter-2 (San Diego, CA). Sixty hours after transfection, the cells were trypsinized and pelleted. Subsequently, the cells were re-plated in the upper chambers of Transwell plates at a density of $50 \times 10^{4} / \mathrm{ml}$ in $200 \mu \mathrm{l}$ of RPMI 1640 . The lower chamber contained $700 \mu \mathrm{l}$ of RPMI 1640 supplemented with $10 \%$ FCS. The subsequent procedures were the same as described above. We performed four individual experiments in which this assay was performed in triplicate.

\section{MMP-2 and VEGF quantification by ELISA}

OVCA cells were seeded into 6-well culture dishes, and incubated in culture medium. After achieving subconfluency, cells were washed with RPMI1640 containing 1\% FCS, and incubated for 48 hours. After incubation, the culture supernatants were tested using MMP-2 ELISA kits (R\&D Systems, Minneapolis, MN) according to the manufacturer's protocol. Experiments were performed in triplicate. In addition, we examined the effect of siRNA transfection on MMP-2 and VEGF expression in ES- 2 cells. ES-2 cells were seeded in 6-well dishes in RPMI1640 containing 10\% FCS. After the cells reached 50\% confluency, the medium was replaced by fresh RPMI 1640 containing $10 \%$ FCS, and transfection of siRNA (si-cont or si-CD13) was performed using a GenePorter-2 (Genlatis, San Diego, CA). Twenty-four hours after transfection, the medium was replaced by fresh RPMI1640 containing 1\% FCS. After 48 hours of incubation, the culture supernatants were collected. The subsequent procedures were the same as described above using MMP-2 and VEGF ELISA kits (R\&D Systems, Minneapolis, MN). 


\section{In vivo studies}

Five-week-old female nude mice (BALB/c) were provided by Japan SLC (Nagoya, Japan). The treatment protocol followed the guidelines for animal experimentation adopted by Nagoya University. HRA or SKOV-3 cells $(5 \times$ $10^{6}$ cells $/ 0.5 \mathrm{ml}$ of medium/mouse) were injected i.p. to examine their peritoneal metastatic potential. The survival time was examined with or without treatment with bestatin.

Intraperitoneal (i.p.) administration of bestatin $(20 \mathrm{mg} /$ kg body weight) was initiated 24 hours after tumor inoculation, and was continued daily for 28 days $(n=7)$. In the control group $(n=7)$, vehicle only was administered in the same manner. Survival time was compared between these two groups. To confirm same results, this experiment was repeated twice.

\section{Statistical analysis}

For data of in vitro and vivo experiments, statistical comparisons among groups were performed by the Student's $t$-test or ANOVA with Bonferroni corrections. Data are expressed as mean $\pm \mathrm{SD}$. $\mathrm{P}<0.05$ was considered significant.

\section{Results \\ APN/CD I 3 expression in OVCA tissues and cell lines}

We first examined APN/CD13 expression in 14 surgically resected OVCA samples. APN/CD13 was predominantly expressed in tumor cells of OVCA tissues, although the intensity of immunohistochemical staining varied from tumor to tumor. (Fig. 1A-D). However, in some tumors, APN/CD13 was also expressed in stromal cells (Fig. 1E). The clinicopathological data of the OVCA tissues examined are summarized in Table 1 . In addition, we found a small number of APN/CD13-positive blood vessels in all OVCA tissues although the vascular density did not seem to be associated with the APN/CD13 expression in the tumor cells. Fig. 2 shows the varying levels of APN/CD13 expression in OVCA cell lines, as measured by FACS analysis. SKOV-3, ES-2 and HEY cells were intensely positive for APN/CD13, while in other cell lines, the level of APN/ CD13 expression was lower, which was consistent with data obtained by enzyme activity analysis (data not shown).

\section{Correlation among APN/CDI 3 expression, migratory potential, MMP-2 expression and cell doubling time}

Table 2 shows the correlation among mean fluorescence intensity of APN/CD13 in FACS, migratory potential, MMP-2 expression, and cell doubling time in these OVCA cell lines. The APN/CD13 expression in these cell lines was positively correlated with their migratory potential and with MMP-2 expression. In contrast, there was no obvious correlation between the expressions of APN/
CD13 and doubling time among these OVCA cell lines. In addition, the morphology of APN/CD13-expressing cell lines tended to show a long spindle/bipolar pattern resembling fibroblasts, in contrast to that of non-APN/ CD13-expressing cell lines, which show an epithelioid pattern.

\section{The effect of bestatin on proliferation and migration of APN/CDI3-expressing OVCA cells in vitro}

To investigate the function of APN/CD13 in OVCA cells, we used bestatin, an APN/CD13 inhibitor, in ES-2 cells, which expressed considerable amounts of APN/CD13. We confirmed that the APN/CD13 activity of ES-2 cells was markedly reduced by bestatin, as demonstrated by reduced absorbance in the enzymatic activity assay $(p<$ 0.001 ) (Fig. 3A). Fig. 3C shows that more than $100 \mu \mathrm{g} / \mathrm{ml}$ of bestatin significantly inhibited the proliferation of ES2 cells. However, bestatin did not influence the proliferation of HRA cells, which expressed a low level of APN/ CD13 (Fig. 3D). We performed the trypan blue dye-exclusion test to evaluate the cytotoxity of the bestatin concentration used. We confirmed that there was no obvious cytotoxic effect of bestatin on HRA cells but that bestatin dose-dependently induced cytotoxicity in ES-2 cells (Fig. 3B).

We also assessed the effect of bestatin on migration in OVCA cells. As shown in Fig. 4, the addition of bestatin significantly inhibited the migration of ES-2 and SKOV-3 cells in the Transwell migration assay in a concentrationdependent manner. In contrast, bestatin did not influence the migration of HRA cells (data not shown).

\section{Effect of inhibition of APN/CDI3 using siRNA on the migration and proliferation of ES-2 cells}

To further confirm the significance of APN/CD13 expression in the cellular function of OVCA, we examined the effect of APN/CD13 silencing using an siRNA system in ES-2 cells. Two siRNAs (si-CD13 and si-CD13-2) that specifically reduced the APN/CD13 protein level were developed. Since the si-CD13 was more efficient for the reduction of $\mathrm{APN} / \mathrm{CD} 13$ expression compared with siCD13-2 (data not shown), we used si-CD13 in the following experiment. Fig. 5A shows Western blot analysis of APN/CD13 expression at 24, 48, and 72 hours post transfection. The APN/CD13 protein level was most efficiently reduced after 72 hours of transfection. At this point, the migratory potential of si-CD13-transfected cells as estimated using the Transwell migration assay was significantly reduced to approximately $30 \%$ of that of the sicont-transfected cells (Fig. 5B). Based on our finding that the migratory potential was downregulated by the silencing of APN/CD13 using siRNA, we next directly examined the effect of APN/CD13 inhibition on the MMP-2 and VEGF expression. The MMP-2 and VEGF expression in the 
A
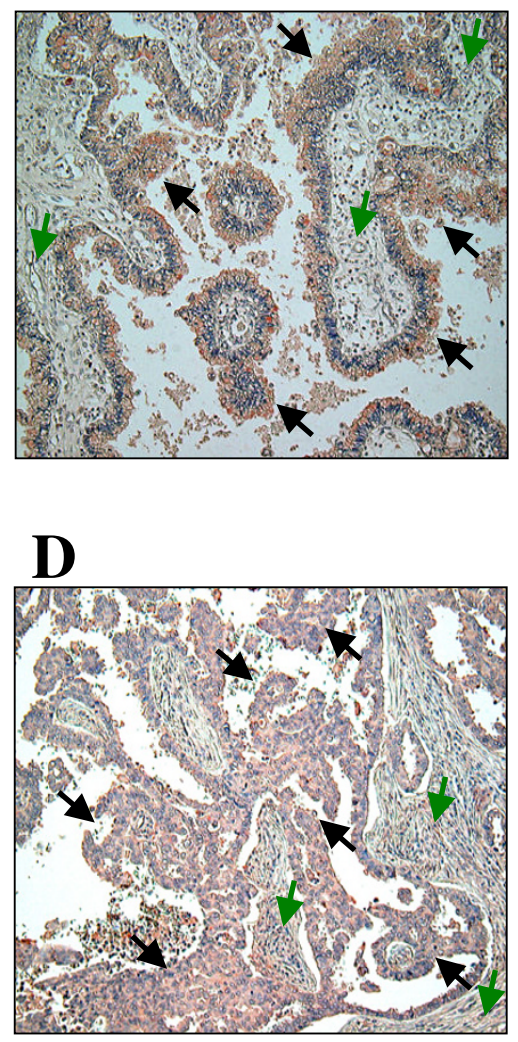

B

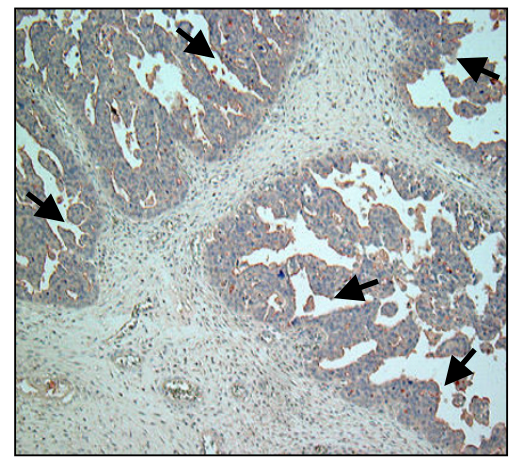

$\mathbf{E}$

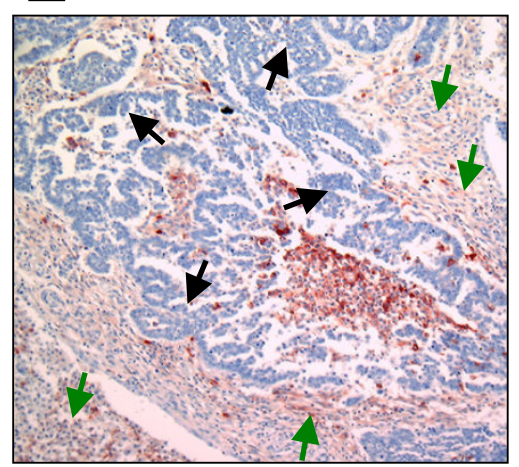

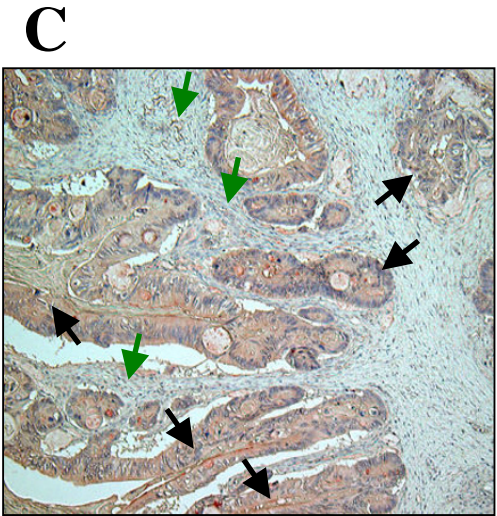

$\mathbf{F}$

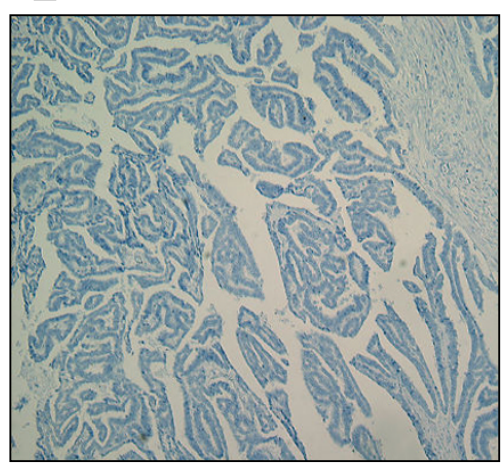

\section{Figure I}

APN/CDI3 expression in OVCA tissues A, B, C, D and E; Immunohistological staining of APN/CDI 3 in surgically resected OVCA tissues of 14 patients representing four different histological types using APN/CDI 3 specific Ab. A-D; APN/CDI 3 was expressed intensely in tumor cells but only slightly in stromal cells. A; clear cell carcinoma (case 7), B; endometrioid adenocarcinoma (case 8), C; mucinous cystadenocarcinoma (case 5), D; serous cystadenocarcinoma (case I2), E; serous cystadenocarcinoma (case 2), APN/CDI3 was intensely expressed in stromal cells but was almost undetectable in tumor cells. F; APN/CDI3negative control of serous cystadenocarcinoma. Black arrows indicate tumor cells expressing APN/CDI3; green arrows indicate stromal cells expressing APN/CDI3.

Table I: Summary of patient characteristics and APN/CD I 3 expression

\begin{tabular}{|c|c|c|c|c|}
\hline Case & PT & Histological type & Tumor grade & $\begin{array}{c}\text { APN/CD I } 3 \text { (tumor } \\
\text { expresion) }\end{array}$ \\
\hline I & Ic & endometrioid & I & $\mathrm{N}$ \\
\hline 2 & $3 c$ & serous & 2 & $\mathrm{~N}$ \\
\hline 3 & $3 c$ & mucinous & 2 & $P$ \\
\hline 4 & $3 c$ & serous & 3 & $\mathrm{~N}$ \\
\hline 5 & la & mucinous & I & $P$ \\
\hline 6 & $3 c$ & mucinous & I & $\mathrm{N}$ \\
\hline 7 & Ic & clear cell & N.D. & $P$ \\
\hline 8 & $2 c$ & endometrioid & 2 & $P$ \\
\hline 9 & Ic & clear cell & N.D. & $P$ \\
\hline 10 & $2 c$ & serous & 3 & $P$ \\
\hline II & Ic & clear cell & N.D. & $\mathrm{N}$ \\
\hline 12 & $3 c$ & serous & 2 & $P$ \\
\hline 13 & Ic & endometrioid & 1 & $\mathrm{~N}$ \\
\hline 14 & la & mucinous & 1 & $\mathrm{~N}$ \\
\hline
\end{tabular}

N.D.: Not determined, N: Negative, P: Positive 

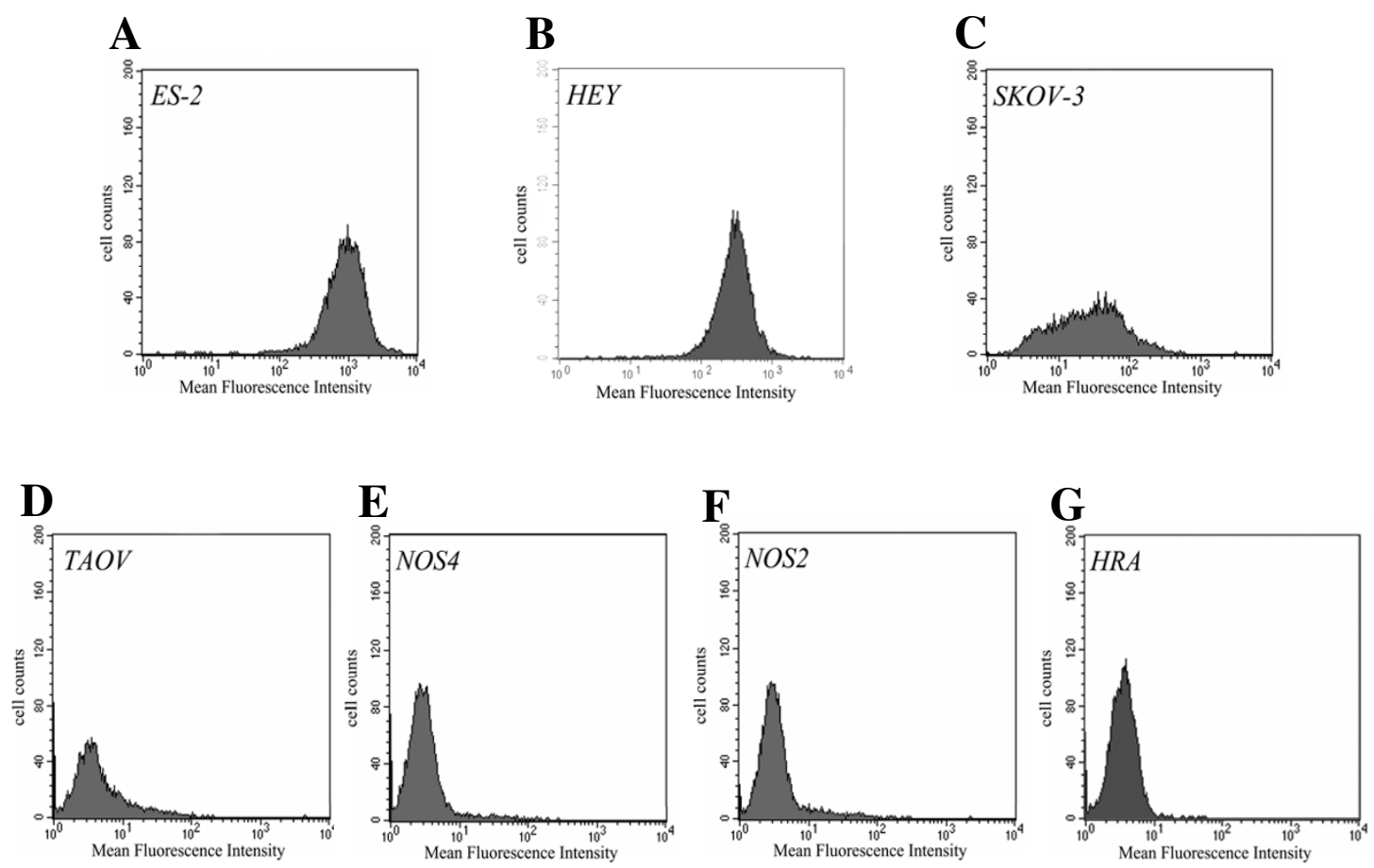

\section{Figure 2}

APN/CDI 3 expression in various OVCA cell lines, as estimated by FACS analysis A; ES-2 cells, B; HEY cells, C; SKOV-3 cells, D; TAOV cells, E; NOS-4 cells, F; NOS-2 cells and G; HRA cells.

Table 2: Correlation among APN/CDI3 expression, migratory potential, and MMP-2 activity in OVCA cell lines

\begin{tabular}{|c|c|c|c|c|c|}
\hline & $\begin{array}{l}\text { APN/CD } 13^{a} \\
\text { expression }\end{array}$ & $\begin{array}{l}\text { Migratory }{ }^{b} \text { cell } \\
\text { counts }\end{array}$ & MMP-2c activity & Doubling time (h) & Morphology \\
\hline ES-2 & 987.3 & $168 \pm 22$ & $34.2 \pm 4.8$ & $18.6 \pm 3.7$ & Fibroblastic \\
\hline HEY & 692.6 & $153 \pm 19$ & $31.5 \pm 5.1$ & $22.3 \pm 2.4$ & Fibroblastic \\
\hline SKOV-3 & 65.2 & $125 \pm 23$ & $23.9 \pm 3.9$ & $18.6 \pm 4.7$ & Fibroblastic \\
\hline HRA & 4.5 & $129 \pm 7$ & $1.9 \pm 0.9$ & $17.4 \pm 2.6$ & Fibroblastic \\
\hline TAOV & 7.4 & $22 \pm 8$ & $3.2 \pm 2.1$ & $20.2 \pm 3.2$ & Epithelioid \\
\hline NOS2 & 5.0 & $11 \pm 4$ & $1.6 \pm 1.2$ & $18.9 \pm 3.7$ & Epithelioid \\
\hline NOS4 & 3.8 & $17 \pm 6$ & $1.7 \pm 0.9$ & $19.4 \pm 3.1$ & Epithelioid \\
\hline
\end{tabular}

a Mean fluorescence intensity by FACS analysis

$b$ By Transwell migration assay $\times 200 \mathrm{HPF}$

c By ELISA assay in conditioned medium $(\mathrm{ng} / \mathrm{ml})$ 
A

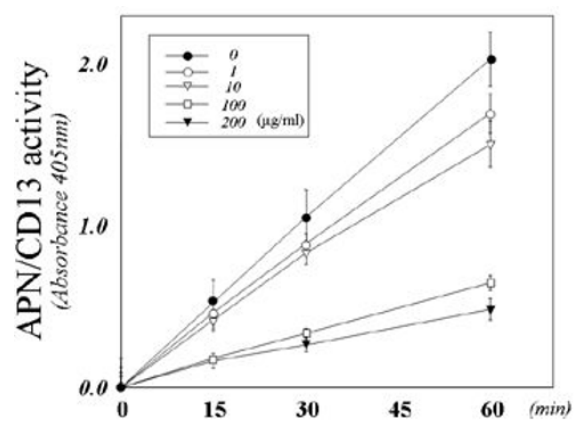

C

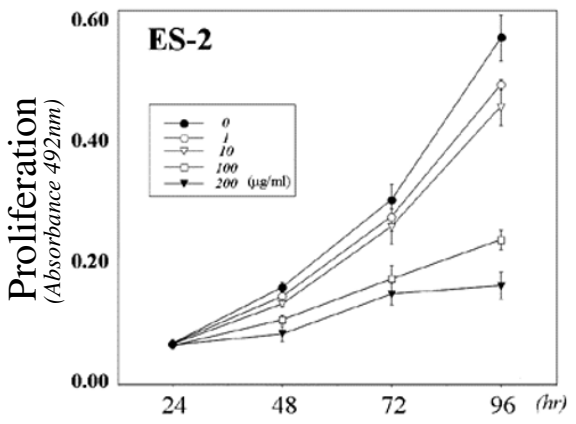

B

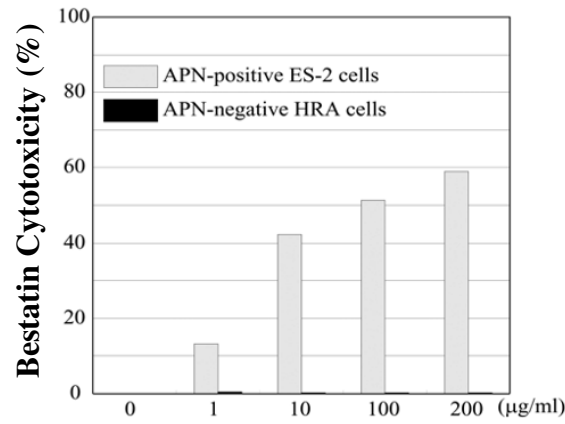

D

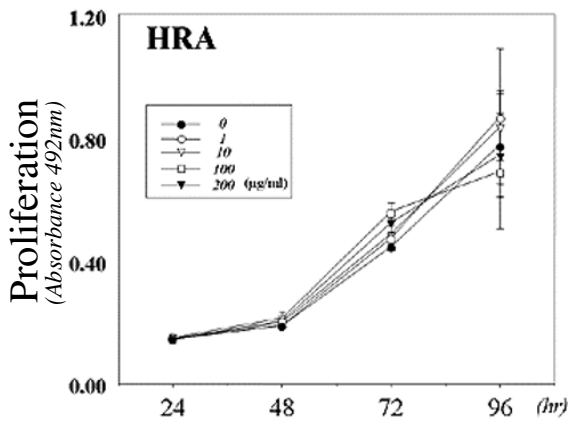

\section{Figure 3}

Differential effects of bestatin on cell proliferation of ES-2 and HRA cells. A; Decrease of APN/CDI 3 enzyme activity by bestatin in a concentration-dependent manner in ES-2 cells, which expressed APN/CDI3. More than $100 \mu \mathrm{g} / \mathrm{ml}$ of bestatin significantly inhibited APN/CDI 3 enzyme activity in ES-2 cells $(p<0.01)$. B; Cytotoxicity of bestatin was evaluated by trypan blue dye-exclusion test for APN/CDI3-positive ES-2 cells and APN/CDI3-negative HRA cells. C; Bestatin dose-dependently suppressed cell proliferation of ES-2 cells, which strongly expressed APN/CDI3. More than $100 \mu g / m l$ of bestatin significantly inhibited the proliferation of ES-2 cells $(p<0.01)$. D; Bestatin exerted no significant effect on the proliferation of HRA cells, which expressed a low level of APN/CDI3.

conditioned medium of si-CD13-transfected ES-2 cells was $20.7 \%$ and $71.1 \%$ lower than that in si-cont-transfected cells, as shown in Figure 6A and 6B, respectively. Furthermore, silencing of APN/CD13 by siRNA resulted in a reduction of the growth rate of ES-2 cells (Fig. 7). The si-cont-transfected cells proliferated at the same rate as the parental ES-2 cells. In contrast, si-CD13-transfected cells could not grow at all.

\section{The effect of bestatin on the peritoneal progression of ovarian carcinoma cells in a nude mice model}

Finally, we tested whether bestatin influenced the peritoneal dissemination or survival of OVCA using nude mice. We previously confirmed that carcinomatous peritonitis was observed approximately 2 or 4 weeks after the inocu- lation of HRA or SKOV-3 cells, respectively, in mice. In the current experiments, daily intraperitoneal administration of bestatin after tumor inoculation was performed. The results showed that in the SKOV-3 cell-inoculated group, treatment with bestatin resulted in decreased peritoneal dissemination and prolonged survival of the mice. On the other hand, in the HRA cell-inoculated group, bestatin did not influence peritoneal dissemination or survival (Fig. 8 and Table 3).

\section{Discussion}

Cell-surface peptidases play a key role in controlling the growth, differentiation and signal transduction of many cellular systems by modulating the activity of peptide factors and regulating their access to receptors [4]. According 
Table 3: The effect of Bestatin on survival time of nude mice

\begin{tabular}{ccc}
\hline Cells & Control-group & Bestatin-group \\
\hline HRA & $13.8 \pm 0.8$ & $15.8 \pm 2.5$ \\
SKOV-3 & $29.0 \pm 3.0$ & $* 36.1 \pm 6.5$ (days) \\
\hline
\end{tabular}

Mean survival days of PBS alone or bestatin treated mice with innoculation of $1.0 \times 10^{7} \mathrm{HRA}$ and SKOV-3 cells, respectively. Data are expressed as the mean \pm SD. *; $p<0.05$

to several other reports, APN/CD13 is involved in the enhanced cell motility and invasive ability of tumor cells, and in the neovascularization of endothelial cells $[5,21$ 23]. In the current study, we focused on the expression and function of APN/CD13 in OVCA. We showed that the morphology of APN/CD13-expressing cell lines tended to show a spindle/bipolar fibroblast-like pattern, which was associated with high invasiveness and enhanced MMP-2 expression. In contrast, cells with low expression of APN/ CD13 showed an epithelioid pattern. This effect of APN/ CD13 on morphology was opposite the effect of DPPIV/ CD26 on OVCA cells shown in our previous report [24]. Based on this result, although APN/CD13 and DPPIV/ CD26 are both cell surface aminopeptidases, their effects on migratory potential and cell morphology are thought to be opposite. Kehlen et al. reported that undifferentiated anaplastic thyroid carcinomas expressed larger amounts of APN/CD13 than differentiated thyroid carcinomas, whereas in contrast, higher levels of DPPIV/CD26 were expressed in follicular and papillary thyroid carcinomas than in undifferentiated anaplastic thyroid carcinomas [23]. Thus, consistent with our results in OVCA, the expression patterns of DPPIV/CD26 and APN/CD13 in thyroid carcinoma were also opposite. Our present data demonstrated that the invasiveness and/or migratory potential are comparatively strong in CD13+/CD26OVCA cells (e.g., ES-2, SKOV-3, and HEY cells); in contrast, these abilities are weak in CD13-/CD26+ OVCA cells (e.g., TAOV, NOS2, and NOS4 cells). We think that there may be a crosstalk between APN/CD13 and DPPIV/CD26 expressions in a variety of cells, and that the expressionbalance between these two molecules may be critical for the cellular invasive potential, although further studies are needed to confirm this. However, HRA cells are CD13-/ CD26-type, and thus their characteristics cannot be explained by the CD13/CD26 balance, and their invasiveness must be based on some other mechanism.

To study the function of APN/CD13 in OVCA, we first used bestatin (Ubenimex), which is a competitive inhibi-

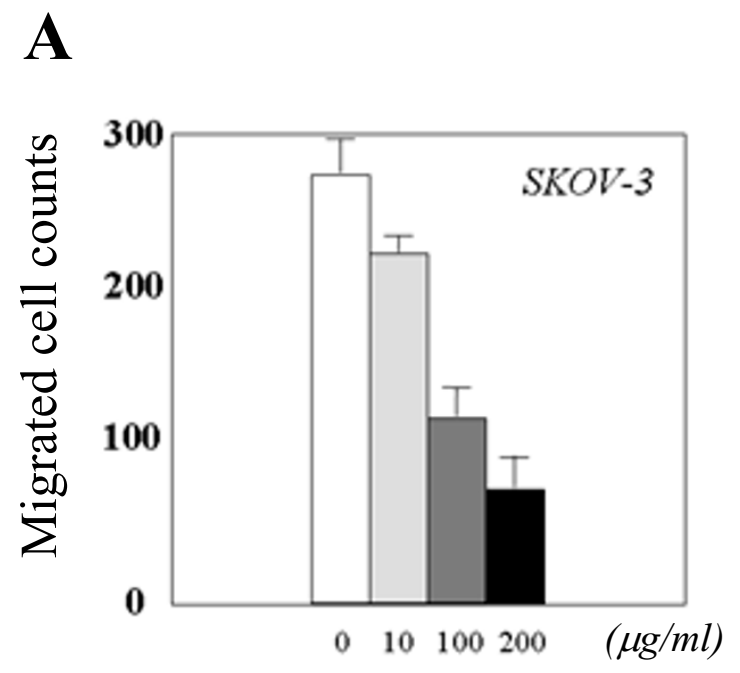

B

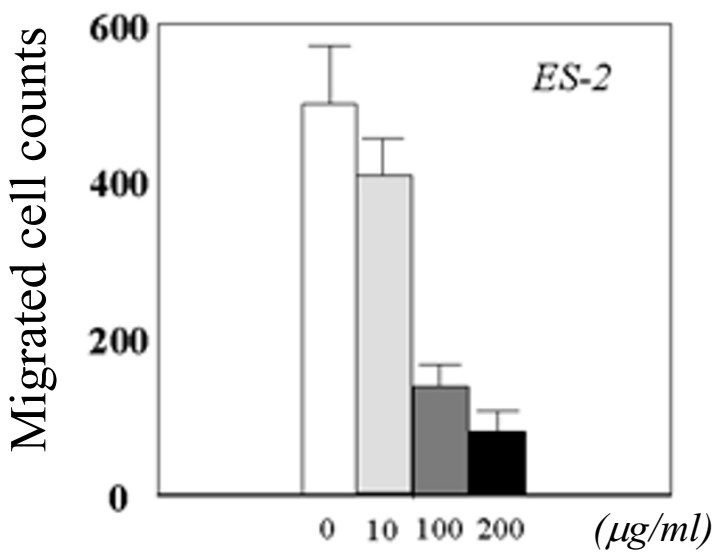

\section{Figure 4}

Bestatin inhibited the cell motility of APN/CDI3-expressing OVCA cells in the Transwell migration assay in a concentrationdependent manner. A: SKOV-3 cells; *; $p<0.0$ I, **; $p<0.00$ I, B; ES-2 cells; *; $p<0.00$ I, **; $p<0.000$ I. 

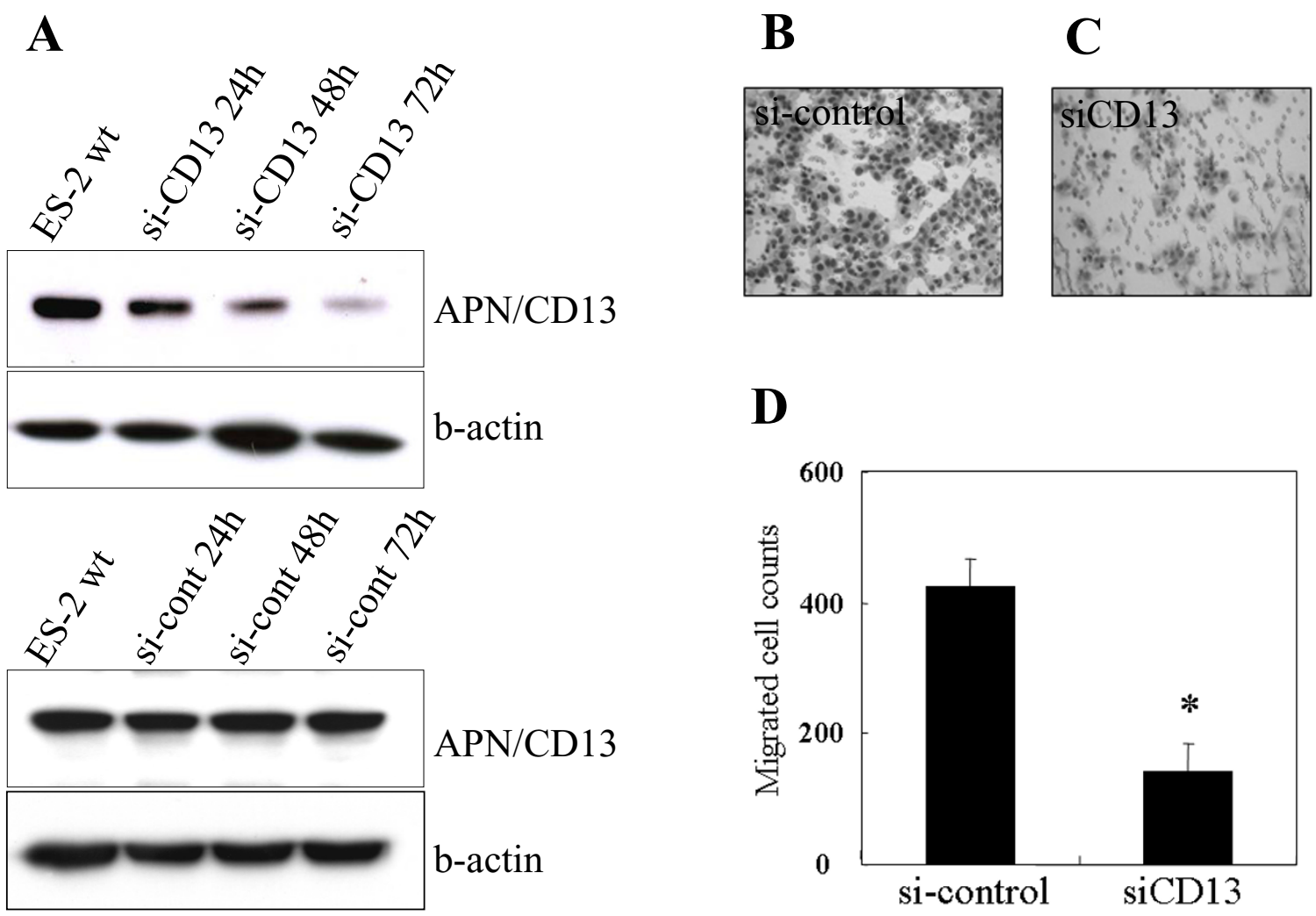

\section{Figure 5}

Suppression of APN/CDI 3 expression by siRNA induced a marked decrease in migratory potential of ES-2 cells. A; Western blot analysis showed a decrease in APN/CDI 3 expression after siRNA transfection. $B$, C; Giemsa staining showing the migration of ES-2 cells transfected with non-specific control siRNA (B; si-cont) or siRNA specific for APN/CDI3 (C; si-CDI3), respectively. D; The level of migration of ES-2 cells transfected with si-CDI3 relative to the control was $33 \%$. Data are expressed as the mean \pm SD $* ; p<0.01$.

tor of APN/CD13 and an antibiotic with inhibitory activity toward some but not all aminopeptidases [25]. Our current findings demonstrated that the suppression of APN/CD13 activity by bestatin in APN/CD13-expressing cells reduced the migratory and proliferative potential as well as the peritoneal dissemination in a mice model, which led to prolonged survival. On the other hand, in OVCA cells with limited expression of APN/CD13, bestatin had no apparent influence on these cell functions related to tumor progression. These results indicated the direct involvement of APN/CD13 in the tumor-progression of OVCA, which was consistent with the finding of previous reports. Considering these results, APN/CD13 activity may play a crucial role in cell motility and the metastasis of OVCA, since APN/CD13 has been suggested to be involved in the degradation of neuropeptides, cytokines, and immunomodulatory peptides, as well as angiotensins [26,27]. Riemann et al. reported that in leukemic cells, APN/CD13 expression may contribute to the malignant phenotype via proteolytically modifying peptides and/or their precursors involved in growth stimulation or retardation [6]. Although bestatin affects the enzymatic activity of APN/CD13, it is not necessarily specific for APN/CD13 and bestatin can directly cause apoptosis in tumor cells besides its ability to inhibit APN/ CD13 activity. Therefore, an siRNA method able to downregulate all functions of APN/CD13 was used in our current in vitro examination. The results showed that silencing of APN/CD13 expression by siRNA induced a marked decrease in the migratory and proliferative potential of OVCA cells with a concurrent down-regulation of MMP-2 and VEGF expression. Chan et al. provided strong evi- 
Fig.6

A

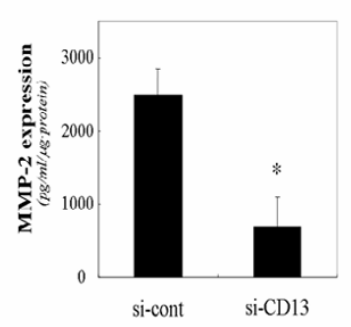

B

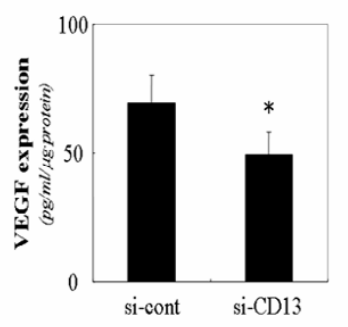

\section{Figure 6}

Decrease of MMP-2 and VEGF expression caused by RNA interference of APN/CDI 3 in ES-2 cells using MMP-2 and VEGF ELISA kit. The MMP-2 and VEGD expression in the conditioned medium of si-CD /3-transfected ES-2 cells was significantly lower than that of si-cont-transfected cells. Data are expressed as the mean $\pm S D, A ;{ }^{*} p<0.00$ I, $B ;{ }^{*} p<0.05$.

dence that APN/CD13 activity was not absolutely required for enhanced migration by demonstrating that expression of a catalytically inactive form of APN/CD13 significantly enhanced the migration of lung adenocarcinoma cells [27]. Taken together, our results also support the notion that APN/CD13 may promote cancer cell migration or metastasis by both aminopeptidase-dependent and aminopeptidase-independent mechanisms.

With regard to the effect of APN/CD13 expression on the progression of OVCA, van Hensbergen et al, previously reported that there was no distinct effect of APN/CD13overexpression on the growth of OVCA cells (IGROV-1 cells) in vitro, but it was associated with decreased invasive ability[28]. In our current study, inhibition of APN/ CD13 by siRNA resulted in a reduction of the growth and invasion of OVCA cells, although there was no obvious correlation between the expression of APN/CD13 and doubling time of various OVCA cells. Unfortunately, we can not explain for this discrepancy at present. However, our assay was based on the inhibition of APN/CD13 expression or its activity using siRNA or bestatin, which was somewhat different from their assays using APN/ CD13-overexpressing transfectants. In addition, the discrepancy may be due to some peculiarity of IGROV-1 cells, because the work of Hensbergen et al, was derived

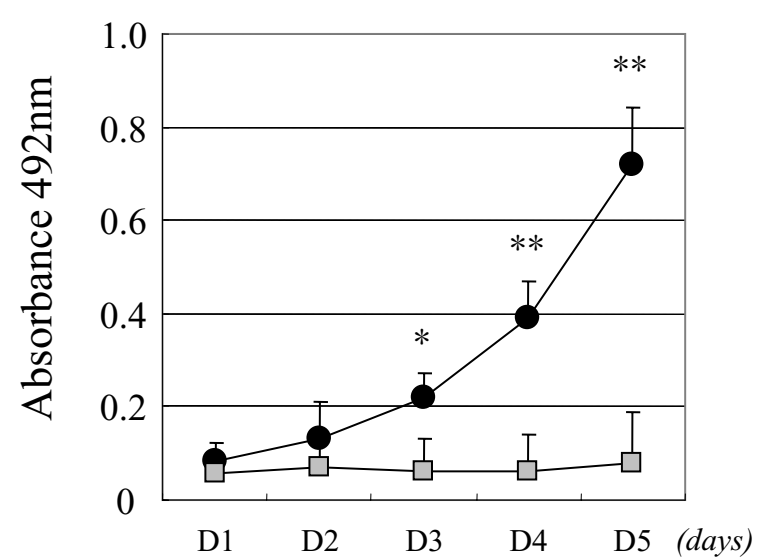

Figure 7

Inhibition of proliferative potential by the transfection of siRNA for APN/CDI 3 in ES-2 cells in a 4-day modified MTT assay. Analysis of the proliferation rate was performed as described in "MATERIALS AND METHODS". si-CDI3-transfected cells showed almost no growth, whereas the si-conttransfected cells grew similarly to the parental ES-2 cells. Closed circles; si-cont-transfected cells, gray squares; si-CDI3transfected cells. Data are expressed as the mean \pm SD of four independent experiments. *; $p<0.0$ I, **; $p<0.00$ I.

from the results using this single cell line. Also, the discrepancy might be due to differences of the expression of several unknown co-factors that facilitate the function of $\mathrm{APN} / \mathrm{CD} 13$. At any rate, to elucidate the mechanistic function of APN/CD13 including these discrepancies, further investigations of APN/CD13 in tumor biology will be essential.

Clinically, several reports have shown that APN/CD13 was correlated with poor prognosis in several malignancies $[9,29]$. Hashida et al. reported that APN/CD13 was involved in cell motility or angiogenesis, and that the expression of this enzyme was associated with poor prognosis for node-positive patients with human colon cancer [9]. According to the recent report from Surowiak et al. [30], there was a slight, although not significant, prognostic difference between patients positive for APN/CD13 expression and those negative for APN/CD13 expression. However, in their study, the majority of the cases were at stage-III (95\%). We think that this could have caused some bias. Namely, in many survival analyses including several clinicopathological factors, stage is an independent prognostic factor. If the influence of stage was very strong, the importance of APN/CD13 expression as a prognostic factor might become weak. Thus, a larger 


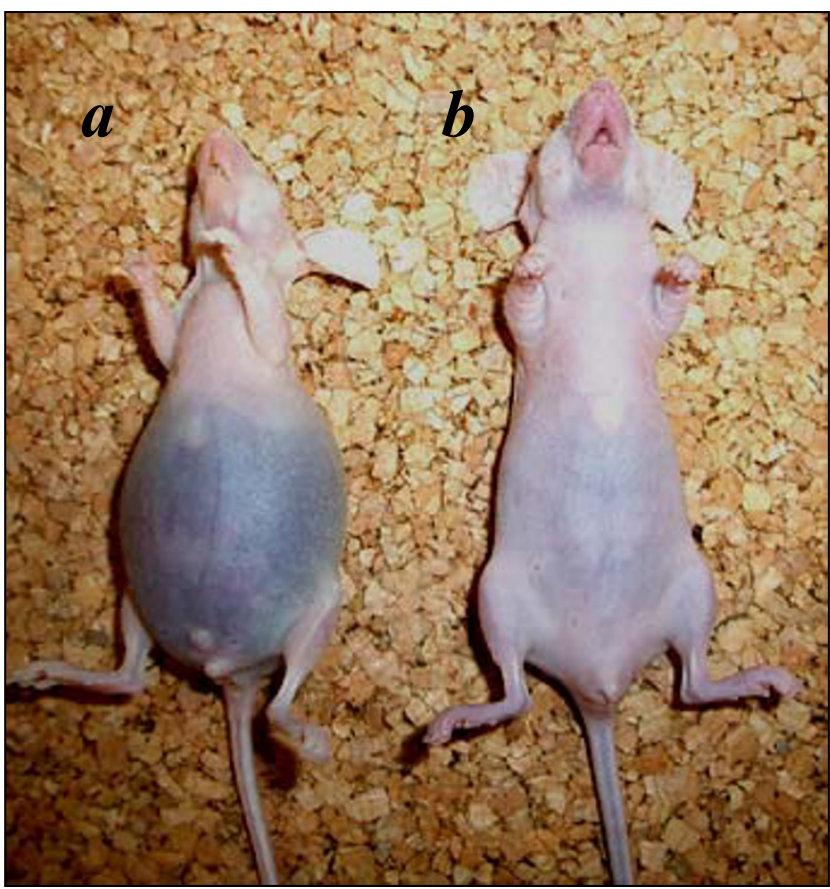

Figure 8

Anti-metastatic effect of bestatin treatment in SKOV-3 cells. A representative example of nude mice 30 days after i.p. inoculation of $1.0 \times 10^{7}$ of SKOV-3 cells with or without subsequent daily treatment with bestatin, showing a mouse treated with PBS alone (a; left) or with bestatin $(20 \mathrm{mg} / \mathrm{kg}$, daily)(b; right). More apparent symptoms of carcinomatous peritonitis were observed in mice treated with PBS alone than in those treated with bestatin. In this experiment, each group consisted of seven mice.

number of patients including early stage (I-II) have to be examined in the next analysis of the prognosis of OVCA.

Considering the body of evidence about APN/CD13, including our current results, APN/CD13 may be a target for treatment of OVCA. If effective, bestatin may be one of the candidate drugs with APN/CD13 as a clinical target, and is already being used clinically for the treatment of leukemia. According to a recent report by Ichinose et al., survival was significantly better for patients with completely resected stage I squamous-cell lung carcinoma who were treated with bestatin as a postoperative adjuvant therapy than for those who received a placebo [31].

\section{Conclusion}

The data obtained in the current investigation demonstrated the expression APN/CD13 in OVCA associated with tumor cell motility, proliferation and metastasis. Therefore, further insights into the mechanism and function of APN/CD13 in tumor biology may be critical, as
APN/CD13 may constitute a potential therapeutic target in a variety of malignancies.

\section{List of abbreviations}

APN: aminopeptidase N, OVCA: ovarian carcinoma.

\section{Competing interests}

The author(s) declare that they have no competing interests.

\section{Authors' contributions}

MT performed all in vitro functional analysis of APN/ CD13 using cell lines and interpreted results and drafted the manuscript. HK performed animal experiment and statistical analysis on the result and designed the expression studies. KS carried out the immunoassays. KN performed the immunohistochemical staining, AN and SM conceived of the study, and participated in its design and coordination. FK supervised all work and aided in the drafting of the manuscript. All authors read and approved the final manuscript.

\section{References}

I. Stange T, Kettmann U, Holzhausen HJ: Immunoelectron microscopic single and double labelling of aminopeptidase N (CD 13) and dipeptidyl peptidase IV (CD 26). Acta Histochem 1996, 98:323-331.

2. Kehlen A, Gohring B, Langner J, Riemann D: Regulation of the expression of aminopeptidase $A$, aminopeptidase $N / C D / 3$ and dipeptidylpeptidase IV/CD26 in renal carcinoma cells and renal tubular epithelial cells by cytokines and CAMP. increasing mediators. Clin Exp Immunol 1998, I I I:435-44 I.

3. Dixon J, Kaklamanis L, Turley H, Hickson ID, Leek RD, Harris AL, Gatter KC: Expression of aminopeptidase-n (CD 13) in normal tissues and malignant neoplasms of epithelial and lymphoid origin. J Clin Pathol 1994, 47:43-47.

4. Shipp MA, Look AT: Hematopoietic differentiation antigens that are membrane-associated enzymes: cutting is the key! Blood 1993, 82: 1052-1070.

5. Bhagwat SV, Lahdenranta J, Giordano R, Arap W, Pasqualini R, Shapiro LH: CDI3/APN is activated by angiogenic signals and is essential for capillary tube formation. Blood 200I, 97:652-659.

6. Riemann D, Kehlen A, Langner J: CDI3--not just a marker in leukemia typing. Immunol Today 1999, 20:83-88.

7. Kehlen A, Egbert I, Thiele K, Fischer K, Riemann D, Langner J: Increased expression of interleukin-8 and aminopeptidase $\mathbf{N}$ by cell-cell contact: interleukin-8 is resistant to degradation by aminopeptidase N/CDI3. Eur Cytokine Netw 200I, 12:316-324

8. Saiki I, Fujii H, Yoneda J, Abe F, Nakajima M, Tsuruo T, Azuma I: Role of aminopeptidase $\mathbf{N}(C D / 3)$ in tumor-cell invasion and extracellular matrix degradation. Int J Cancer 1993, 54:137-143.

9. Hashida $\mathrm{H}$, Takabayashi A, Kanai M, Adachi M, Kondo K, Kohno N, Yamaoka $\mathrm{Y}$, Miyake M: Aminopeptidase $\mathbf{N}$ is involved in cell motility and angiogenesis: its clinical significance in human colon cancer. Gastroenterology 2002, I 22:376-386.

10. Kido A, Krueger S, Haeckel C, Roessner A: Inhibitory effect of antisense aminopeptidase N (APN/CDI3) cDNA transfection on the invasive potential of osteosarcoma cells. Clin Exp Metastasis 2003, 20:585-592.

II. Kido A, Krueger S, Haeckel C, Roessner A: Possible contribution of aminopeptidase $\mathbf{N}$ (APN/CDI3) to invasive potential enhanced by interleukin- 6 and soluble interleukin- 6 receptor in human osteosarcoma cell lines. Clin Exp Metastasis 1999, I 7:857-863.

12. Wex T, Lendeckel U, Reinhold D, Kahne T, Arndt M, Frank K, Ansorge S: Antisense-mediated inhibition of aminopeptidase 
N (CDI3) markedly decreases growth rates of hematopoietic tumour cells. Adv Exp Med Biol 1997, 42 1:67-73.

13. Curnis F, Arrigoni G, Sacchi A, Fischetti L, Arap W, Pasqualini R, Corti A: Differential binding of drugs containing the NGR motif to CDI 3 isoforms in tumor vessels, epithelia, and myeloid cells. Cancer Res 2002, 62:867-874.

14. Pasqualini R, Koivunen E, Kain R, Lahdenranta J, Sakamoto M, Stryhn A, Ashmun RA, Shapiro LH, Arap W, Ruoslahti E: Aminopeptidase $\mathbf{N}$ is a receptor for tumor-homing peptides and a target for inhibiting angiogenesis. Cancer Res 2000, 60:722-727.

15. Mishima Y, Matsumoto-Mishima Y, Terui Y, Katsuyama M, Yamada M, Mori M, Ishizaka $Y$, Ikeda K, Watanabe J, Mizunuma N, Hayasawa $\mathrm{H}$ Hatake K: Leukemic cell-surface $C D$ I 3/aminopeptidase $\mathbf{N}$ and resistance to apoptosis mediated by endothelial cells. J Natl Cancer Inst 2002, 94: 1020-1028.

16. Brun JL, Feyler A, Chene G, Saurel J, Brun G, Hocke C: Long-term results and prognostic factors in patients with epithelial ovarian cancer. Gynecol Oncol 2000, 78:2I-27.

17. Kikkawa F, Matsuzawa K, Arii Y, Kawai M, Kobayashi I, Nakashima N, Mizutani S: Randomized trial of cisplatin and carboplatin versus cisplatin, vinblastine and bleomycin in ovarian cancer. Gynecol Obstet Invest 2000, 50:269-274.

18. Nishikawa M, Itakura A, Ito M, Takeuchi M, Sato Y, Kajiyama H, Mizutani S, Kikkawa F: Changes in placental dipeptidyl peptidase IV in preeclampsia with intrauterine growth restriction. Horm Metab Res 2005, 37:408-4I3.

19. Kajiyama H, Kikkawa F, Suzuki T, Shibata K, Ino K, Mizutani S: Prolonged survival and decreased invasive activity attributable to dipeptidyl peptidase IV overexpression in ovarian carcinoma. Cancer Res 2002, 62:2753-2757.

20. Uslu R, Borsellino N, Frost P, Garban $\mathrm{H}, \mathrm{Ng} C P$, Mizutani $Y$, Belldegrun $A$, Bonavida $B$ : Chemosensitization of human prostate carcinoma cell lines to anti-fas-mediated cytotoxicity and apoptosis. Clin Cancer Res 1997, 3:963-972.

21. Bhagwat SV, Petrovic N, Okamoto Y, Shapiro LH: The angiogenic regulator CDI3/APN is a transcriptional target of Ras signaling pathways in endothelial morphogenesis. Blood 2003, I01:1818-1826.

22. Petrovic N, Bhagwat SV, Ratzan WJ, Ostrowski MC, Shapiro LH: CDI3/APN transcription is induced by RAS/MAPK-mediated phosphorylation of Ets- 2 in activated endothelial cells. J Biol Chem 2003, 278:49358-49368.

23. Kehlen A, Lendeckel U, Dralle H, Langner J, Hoang-Vu C: Biological significance of aminopeptidase N/CDI3 in thyroid carcinomas. Cancer Res 2003, 63:8500-8506.

24. Kikkawa F, Shibata K, Ino K, Nomura S, Kajiyama H, Suzuki T, Kawai M, Mizutani S: Preoperative findings in non-gynecologic carcinomas metastasizing to the ovaries. Gynecol Obstet Invest 2002 54:221-227.

25. Scornik OA, Botbol V: Bestatin as an experimental tool in mammals. Curr Drug Metab 200I, 2:67-85.

26. Lendeckel U, Arndt M, Frank K, Wex T, Ansorge S: Role of alanyl aminopeptidase in growth and function of human $T$ cells (review). Int J Mol Med 1999, 4:17-27.

27. Chang YW, Chen SC, Cheng EC, Ko YP, Lin YC, Kao YR, Tsay YG, Yang PC, Wu CW, Roffler SR: CDI3 (aminopeptidase N) can associate with tumor-associated antigen $\mathrm{L} 6$ and enhance the motility of human lung cancer cells. Int J Cancer 2005, I I 6:243-252.

28. van Hensbergen Y, Broxterman HJ, Rana S, van Diest PJ, Duyndam MC, Hoekman K, Pinedo HM, Boven E: Reduced growth, increased vascular area, and reduced response to cisplatin in CD I3-overexpressing human ovarian cancer xenografts. Clin Cancer Res 2004, 10: I 180- I | 9 |.

29. Ikeda N, Nakajima $Y$, Tokuhara T, Hattori N, Sho M, Kanehiro $H$, Miyake M: Clinical significance of aminopeptidase N/CDI3 expression in human pancreatic carcinoma. Clin Cancer Res 2003, 9:1503-1508.

30. Surowiak P, Drag M, Materna V, Suchocki S, Grzywa R, Spaczynski M, Dietel M, Oleksyszyn J, Zabel M, Lage H: Expression of aminopeptidase N/CDI 3 in human ovarian cancers. Int J Gynecol Cancer 2006, 16:1783-1788.

31. Ichinose $Y$, Genka K, Koike T, Kato H, Watanabe $Y$, Mori T, lioka $S$, Sakuma A, Ohta M: Randomized double-blind placebo-controlled trial of bestatin in patients with resected stage I squamous-cell lung carcinoma. J Natl Cancer Inst 2003, 95:605-6I0.

\section{Pre-publication history}

The pre-publication history for this paper can be accessed here:

http://www.biomedcentral.com/1471-2407/7/140/pre pub 